\title{
A Research on Kyphoplasty Instrument of Memory Alloy Wire Netting Ball
}

\author{
Xinyu Zhang ${ }^{1, \text { a }}$ \\ P.E Department, Harbin University of Science and Technology, Harbin, Heilongjiang, China
}

Keywords: memory alloy; netting ball; kyphoplasty; biomechanics; feasibility

\begin{abstract}
Purpose of research: developing a kyphoplasty instrument of memory alloy wire netting ball and discussing the possible treatment of osteoporotic vertebral compression fracture. Method: The application of Ni-Ti alloy hyperelastic wire netting ball, the application of the equipment which can stretch and compress the wire netting to conduct kyphoplasty and the test of the peak load. Result: the diameter of the stretched memory alloy wire netting ball is $4.5 \mathrm{~mm}$; the diameter can be $15 \mathrm{~mm}$ thanks to kyphoplasty instrument which restore the shape of ball. The peak load could reach 169.39 by the intense open force according to the biomechanical tests. Conclusion: the kyphoplasty instrument of memory alloy wire netting ball can be used in the treatment of osteoporotic vertebral compression fracture while its clinical application needs more researches.
\end{abstract}

\section{Introduction}

With increasing aging population the patient of vertebral compression fracture (VCF) which can cause lumbar and back ache become more, which lower the people's life quality. [1] Kyphoplasty, a new treatment of VCF in recent years could prevent the leakage of the filling material, stabilize the fracture, recover the mechanical strength of centrum, prevent its compression and relieve the pain. [2] This research aims to develop a hyperelastic kyphoplasty instrument of memory alloy wire netting ball by using hyperelastic Ni-Ti alloy and discuss the possible treatment of vertebral compression fracture by pedicle.

\section{Material and Method}

\subsection{The design of kyphoplasty instrument of memory alloy wire netting ball}

A wire netting ball equipment (picture 1) which could be stretched and compressed is designed with a hyperelastic Ni-Ti alloy wire netting ball with a diameter of $15 \mathrm{~mm}$, a length of $20 \mathrm{~mm}$ and rhombic mesh whose side length is $2 \mathrm{~mm}$ which is British royal aristocracy's brand Slazenger's product with a diameter of 0.38 . One side of the wire netting ball is fixed on the inner core while its other side is fixed on the outer sleeve. The wire netting ball can be stretched and compressed by rolling the handle at the end of them. 
Picture 1. wire netting ball

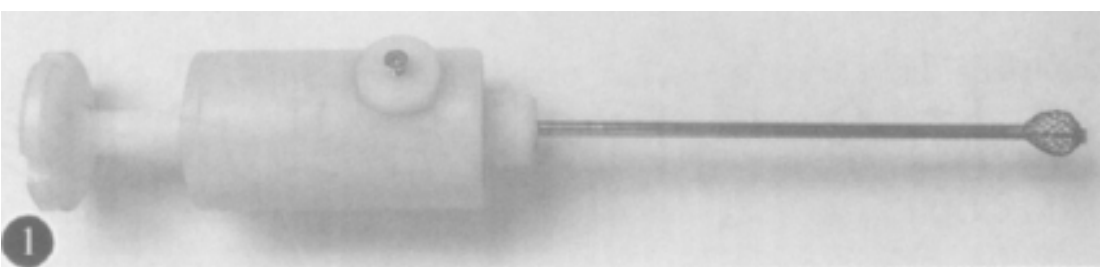

\subsection{The method of kyphoplasty on transpedicular}

The lumbar spine specimen from a frozen dead aged woman is used for the experiment. The specimen's surrounding soft tissues are removed after it is unfrozen. The osteointroducer whose diameter is $5 \mathrm{~mm}$ is placed in the vertebral body by the guiding needle which enters the vertebral body through pedicle. The stretched wire netting ball enters the vertebral body through osteointroducer and its position is measured by X- ray. The wire netting ball recovers its ball shape due to the rolling of the handle at the end of the kyphoplasty equipment and the position and the shape of the wire netting ball is also measured by X-ray.

\subsection{The biomechanics measurement of wire netting ball}

The wire netting ball is placed horizontally in a metal ring which is $5 \mathrm{~mm}$ high and whose inner diameter is $15 \mathrm{~mm}$ and next it is put on the base of the MTS 858Mini. Bionix 2 biomechanics testing machine in which pressure is added vertically at a speed of $0.2 \mathrm{~cm} / \mathrm{min}$ within the distance of $10 \mathrm{~mm}$ and is removed at the same speed. The changing curve of the wire netting ball is recorded in this process.

\section{Conclusions}

The Ni-Ti alloy wire netting ball is hyperelastic so its diameter of the wire netting ball is $4.5 \mathrm{~mm}$ after being stretched by the Kyphoplasty equipment (picture 2a) and the diameter is $15 \mathrm{~mm}$ after the wire netting ball restore the ball shape due to the compression of the outer cylinder of the kyphoplasty instrument.

Picture 2a. the diameter of the wire netting ball is $4.5 \mathrm{~mm}$ after being stretched

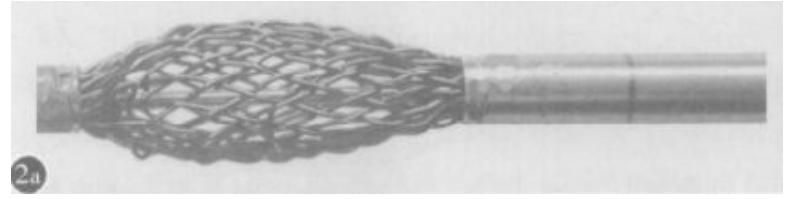

Picture 2b. the diameter of the wire netting ball is $15 \mathrm{~mm}$ after being compressed

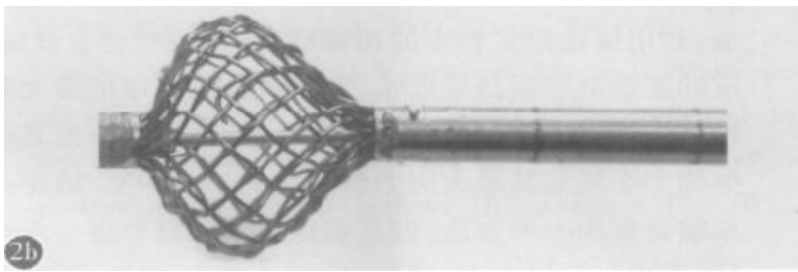


The wire netting ball is inserted into the pipe which is inserted into the vertebra through the pedicle after being stretched (picture $3 \mathrm{~b}$ ) then it restores the ball shape due to the compression of the outer cylinder with the rolling of the handler at the end of Kyphoplasty equipment. (picture 3c、d).

Picture 3a. the pipe inserted into vertebra through pedicle

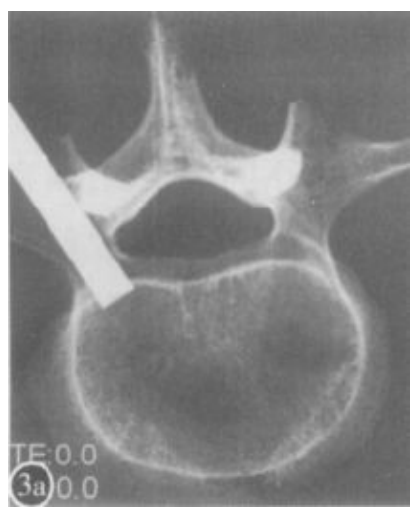

Picture 3b. the wire netting ball inserted into the vertebral through pedicle

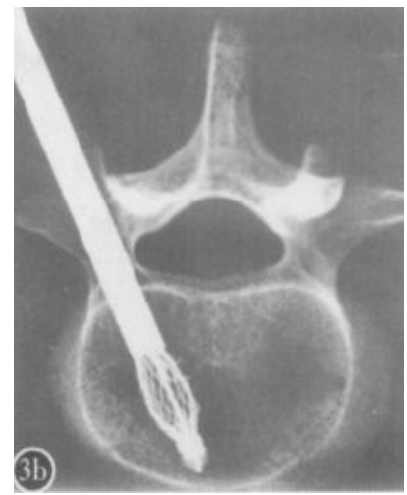

Picture 3c、d. wire netting ball which restore the ball shape with the compression of the outer cylinder
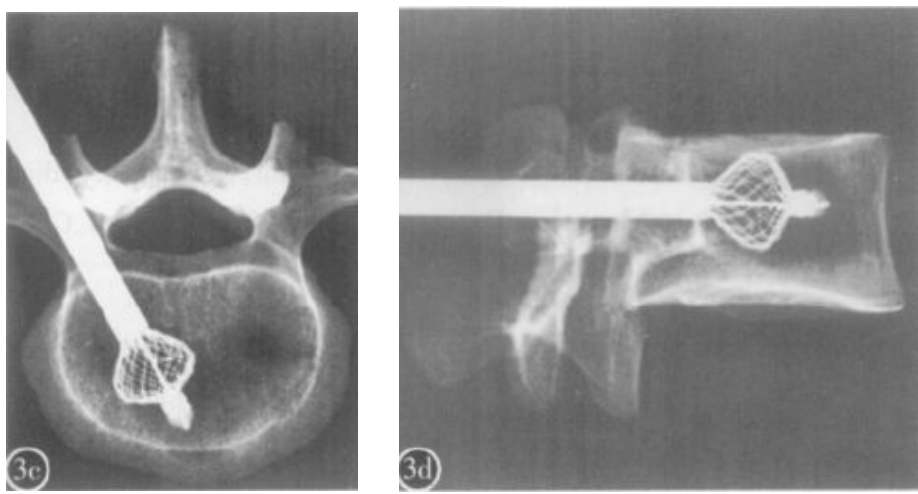

The maximum bearable force can reach $162.39 \mathrm{~N}$ according to the experiment of adding force to the wire netting ball. Due to the hyperelastic Ni-Ti alloy the wire netting ball has the same maximum bearable force in the numerable texts. 


\section{DISCUSSION}

\subsection{Minimally invasive treatment of VCF}

Mobidity of VCF which can cause ache of the patient and limit their movement increases while more and more people become old. Due to the inefficiency of the traditional conservative treatment vertebroplasty or kyphoplasty is used to treat VCF in recent years. In vertebroplasty bone cements are injected into the centrum through the skin or they are injected after the centrum is opened by the balloon in order to stabilize the fracture, recover the mechanical strength and relieve the ache. Comparing with the fulfillment of bone cements in vertebroplasty balloon is applied to open the centrum after successful puncture to restore the height of centrum, lessen the possibility of kyphosis, in kyphoplasty form cavity in centrum, fill bone cement with low pressure and prevent the leak of bone cement. But there are several disadvantages such in kyphoplasty such as the too complicated technology, long hours of surgery and its high expenses. Furthermore the balloon always moves to the other area and lead to the failure of opening the fracture part and it is also always punctured by the marginal part of the bone. This research aims to design a new wire netting ball kyphoplasty instrument and develop a new minimally invasive treatment of VCF.

\subsection{The principle of memorial wire netting ball kyphoplsty instrument}

$\mathrm{Ni}$-Ti memorial alloy is made of $\mathrm{Ni}$ and $\mathrm{Ti}$ according to a proper proportion. It has a lot of advantages such as its good memory of the shape, hyperelastic feature, low magnetism, fatigue endurance, attrition endurance and well biocompatibility. The elasticity of Ni-Ti alloy which is different from the one caused by the atoms variations is called hyperelasticity. The elasticity is as 10 times as the common material such as stainless steel, copper alloy spring wire and so on. Therefore the Ni-Ti alloy can be applied extensively in stabilizing the fracture, orthopedic surgery of scoliosis and the treatment of avascular necrosis of femoral head. Yan Wang and so on use wire netting ball support which is made of Ni-Ti alloy to treat the adult avascular necrosis of femoral head by increasing the mechanic support of subchondral bone in weight-bearing area and lower the local stress, which has a great benefits in repair and reconstruction of the area of avascular necrosis of femoral head and has a better effect. The Ni-Ti alloy wire netting ball has the same hyperelasticity so the reinforcing material can be filled in the cavity in compressed fracture centrum which is opened by the force caused by compressing the netting ball which enter the centrum through the pipe by stretching equipment the into ball shape.

\section{3 the feasibility of the treatment of the VCF by the wire netting ball kyphoplsty}

The kyphoplasty wire netting ball in this research is hyperelastic and it is feasible to treat VCF with minimal invasive surgery. The maximum bearable force could be $162.39 \mathrm{~N}$ according to the biomechanics text. The balloon don't move to the other area in the process of conversion into ball shape and can open the centrum of fracture without braked by the sharp margin of the bone. The wire netting ball could retreat from the centrum after opening it then the centrum strengthening material is filled in with low pressure, which can lower the possibility of complication such as leakage and embolism. Wire netting ball has a better mechanical property and biocompatibility, which could form armoured concrete structure with bone cement which could make the filling material in the compressed fracture centrum having a better biomechanics force.

The research prove that the kyphoplasty wire netting ball is suitable for the treatment of compressed bone fracture caused by VCF but the efficiency and safety of this treatment need a further study clinically. 


\section{References}

1. Yan Wang: the minimal invasive treatment of the compressed centrum fracture caused by VCF, p.995- 998 (Chinese Journal of Orthopaedic Trauma, China 2004, 6(9))

2. Keyna MaoBaowei Liu, Yan Wan and so on: The influence of fulfillment of carbonated hydroxyapatite cement on the centrum height and ache of the fracture patient with VCF., p. 9899(Journal of Chinese Clinical Recovery, China 2005, 9(2))

3. Yan Wang, Jifang wang, Shibi Lu and so on: The treatment of adult ischemic necrosis of femoral head by wire netting support, p. 295-298(Chinese Journal of Orthopaedic Trauma, China 2000, 20(5))

4. Jarvik JG, Kallmes DF, Mirza SK: Vertebroplasty learning more,but not enough, p. 14871489(Spine, 2003, 28(14))

5. Hide IG, Gangi A.Percutaneous vertebroplasty: history, technique and current perspectives, p. 461- 467 (Clin Radiol, 2004, 59(6))

6. Rao RD, Singrakhia MD: Current concepts review painful osteo-porotic vertebral fracture, pathogenesis evaluation, and roles of vertebroplasty and kyphoplasty in its management, p. 2010- 2022 (J Bone Joint Surg(Am), 2003, 85(10))

7. Carrino JA, Chan R, Vaccaro AR.Vertebral augmentation: ver-tebroplasty and kyphoplasty, p. 68- 84 (Semin Roentgeno, 2004, 39(1))

8. Yu SW, Chen WJ, Lin WC, et al: Serious pyogenic spondylitis following vertebroplasty: a case report, p. 209-211(Spine, 2004, 29(10))

9. Wever DJ, Elstrodt JA, Veldhuizen AG, et al: Scoliosis correc-tion with shape - memory metal: results of an experimental study, p. 100- 106 (Eur Spine J, 2002, 11(2))

10. Braun JT, Ogilvie JW, Akyuz E, et al: Fusionless scoliosis cor-rection using a shape memory alloy staple in the anterior thoracic spine of the immature goat, p. 1980- 1989 (Spine, 2004, 29 (18))

11. Yan Wang, Dewei Zhao, Jifang Wang and so on: The preliminary report of the treatment of adult avascular necrosis of femoral head by $\mathrm{Ni}$ - Ti alloy wire netting ball, p. 579-581 (Chinese Journal of Surgery, 1998, 36(10)) 